\title{
Las panacas y el poder en el Tahuantinsuyo
}

Les panaca et le pouvoir dans le Tahuantinsuyo

The panacas and power in Tahuantinsuyo

Francisco Hernández Astete

\section{(2) OpenEdition}

Journals

Edición electrónica

URL: http://journals.openedition.org/bifea/3282

DOI: $10.4000 /$ bifea.3282

ISSN: 2076-5827

Editor

Institut Français d'Études Andines

Edición impresa

Fecha de publicación: 1 abril 2008

Paginación: 29-45

ISSN: 0303-7495

Referencia electrónica

Francisco Hernández Astete, "Las panacas y el poder en el Tahuantinsuyo », Bulletin de l'Institut français d'études andines [En línea], 37 (1) | 2008, Publicado el 01 octubre 2008, consultado el 10 diciembre 2020. URL : http://journals.openedition.org/bifea/3282 ; DOI : https://doi.org/10.4000/bifea. 3282

\section{(c) $(1) \odot$}

Les contenus du Bulletin de l'Institut français d'études andines sont mis à disposition selon les termes de la licence Creative Commons Attribution - Pas d'Utilisation Commerciale - Pas de Modification 4.0 International. 


\title{
Las panacas y el poder en el Tahuantinsuyo
}

\author{
Francisco Hernández Astete*
}

\section{Resumen}

Partiendo del análisis de la bibliografía sobre los incas y de las fuentes coloniales disponibles, se estudia la categoría de panaca. En este sentido, se discute la división tradicional de la nobleza cuzqueña en 10 ó 12 panacas, a partir de la relectura de estas fuentes y del análisis del significado del término panaca. Se propone que el Cuzco incaico estuvo dividido en dos grupos de nobles: una nobleza principal, llamada Cápac Aillu, y una nobleza secundaria, llamada Hatun Aillu. Cápac Aillu estuvo integrado por los descendientes de los Incas en mujeres nobles (pana), y Hatun Aillu, por los descendientes de los Incas en mujeres no nobles provenientes de las élites locales. Finalmente, se concluye que las llamadas panacas fueron, en realidad, los aillus que integraban a los descendientes de las mujeres nobles identificadas como pana.

Palabras clave: Cuzco, Incas, autoridad, panacas, Perú prehispánico, historiografía

\section{Les panaca et le pouvoir dans le Tahuantinsuyo}

\section{Résumé}

Cet article examine la catégorie des panaca : à partir de la bibliographie sur les Incas, de la relecture des sources coloniales disponibles et de la clarification de ce terme, l'analyse porte sur la division traditionnelle de la noblesse de Cuzco en dix ou douze panaca. L'hypothèse est que le Cuzco incaïque était divisé en deux groupes de nobles : une noblesse principale, appelée Capac Aillu, et une noblesse secondaire ou Hatun Aillu. Capac Aillu était constitué par les descendants des Incas et de femmes nobles (pana) tandis que Hatun Aillu, comprenait ceux qui étaient issus de femmes roturiaires provenant des élites locales. Finalement, on conclut que ces panaca correspondaient en réalité aux aillus composés des descendants de ces femmes nobles indentifiées comme pana.

Mots clés : Cuzco, Incas, autorité, panacas, Pérou préhispanique, historiographie 


\title{
The panacas and power in Tahuantinsuyo
}

\begin{abstract}
This analysis of the word and category «panaca» is examined based on the bibliography on the Incas and the available colonial sources. The traditional division of the Cuzqueño nobility in 10 or 12 panacas is disputed and it is proposed that the elite of the Cuzco of was divided into two groups of nobles: a main nobility called Cápac Aillu, and a secondary one called Hatun Aillu. Cápac Aillu was made up of the descendants of the Incas by noble women (pana), and Hatun Aillu included the descendants of the incas by other women belonging to the local elites. It is concluded that the so-called panacas were, in fact, the aillus made up of the descendants of the noble women identified as «pana».
\end{abstract}

Key words: Cuzco, Incas, authority, panacas, Peru prehispanic, historiography

\section{INTRODUCCIÓN}

Las fuentes coloniales contienen referencias lo suficientemente claras sobre la élite incaica por lo que no es posible dudar acerca de su autoridad en el mundo prehispánico y de su evidente incorporación a la sociedad colonial. Sin embargo, estas mismas fuentes presentan información contradictoria acerca de su estructura y funcionamiento. Por esta razón, desde hace más de cuarenta años existen debates académicos sobre la organización y sustento de la élite incaica. En ese sentido, tanto la distinción hecha por los cronistas entre «nobleza de sangre» y «nobleza de privilegio»1, como la composición misma de la primera, han sido abordadas desde diversas perspectivas teóricas por lo que hoy disponemos de propuestas divergentes sobre el tema2.

De hecho, una de las primeras distinciones al interior de la más alta nobleza cuzqueña es la que diferencia aillus de panacas. Al respecto, existe cierto consenso en la bibliografía que se refiere a los incas. Se asume que los aillus correspondían, dentro de la élite incaica, a un nivel jerárquico inferior al de las panacas y se les identifica como aliados de los incas desde su llegada al Cuzco, asumiendo que su presencia en el área se pierde en la información mítica. Por su parte, las panacas incaicas, de mayor jerarquía que los aillus, se vincularían directamente con los descendientes de los Incas reinantes e integrarían la más alta nobleza cuzqueña. Es necesario mencionar, sin embargo, que tanto la interpretación asignada a las panacas como la que se otorga a los aillus cuzqueños merece aún el interés de los investigadores debido a que los argumentos sobre los que se ha construido la información acerca de sus distinciones y definiciones son bastante laxos y la propia historiografía presenta huellas de estas contradicciones. En ese sentido, a modo de ejemplo, en opinión de John Rowe (Rowe, 2003: 45 y ss.), aillu y panaca probablemente fueron sinónimos antes del siglo XVI. En esta oportunidad nos interesará hacer algunas precisiones acerca de las

1 La llamada nobleza de privilegio estaría asociada con las élites locales. Aparentemente su reconocimiento por parte de los incas marcaría diferencias entre ellos asociadas con el modo en que se incorporaron al Tahuantinsuyo. Así, los privilegios de los curacas, o hatun curacas, debieron depender de si su incorporación al llamado Tahuantinsuyo fue producto de una conquista o de una negociación. Evidentemente, el tipo de negociación, generaba una más o menos provechosa alianza con los cuzqueños.

2 Para mayores detalles puede consultarse: Bravo, 1986; 1992; Duviols, 1980; Pärssinen, 2003; Pease, 1992; Regalado, 1996a; Rostworowski, 1983; Rowe, 1985; Zuidema, 1995 [1964]; Ziólkowski, 1997. 
panacas, principalmente acerca de la manera cómo se ha construido historiográficamente el contenido de esta categoría.

Como se ha mencionado, existe consenso en la historiografía acerca de que la nobleza cuzqueña estuvo organizada en panacas, las mismas que son definidas como grupos de parentesco extendido formados a partir de un Sapan Inca3 y que estaban integradas por todos sus descendientes, exceptuando a aquel que quedaba convertido en gobernante, quien debía abandonar la panaca paterna y formar la propia. De hecho, los españoles del siglo XVI encontraron descendientes de los Incas, muchos de los cuales se incorporaron a la vida del Perú colonial. A estos personajes los investigadores contemporáneos suelen identificarlos, casi automáticamente, como «militantes» de alguna de las panacas incaicas a partir del Inca del que afirman descender. Así, aunque hay algunas discrepancias sobre la existencia de determinadas panacas, existe también acuerdo sobre la presencia de otras de modo que, aparentemente, solo quedaría en discusión la existencia de la panaca fundada por Huáscar y la de algunas otras que María Rostworowski llamó panacas añadidas (Rostworowski, 1983: 141 y ss.)

Casi siempre sobre la información que presenta Pedro Sarmiento de Gamboa, los investigadores reconocen como oficial esta relación de panacas, integrada, como se ha mencionado, por todos los descendientes de cada Inca reinante. Las cinco primeras identificadas con Rurin $\mathrm{CuzCO}^{4}$ y las siguientes con la parcialidad de Hanan Cuzco:

\begin{tabular}{|l|l|}
\hline \multicolumn{1}{|c|}{ Fundador } & \multicolumn{1}{c|}{ Panaca } \\
\hline Manco Cápac & Chima Panaca Aillu \\
\hline Sinchi Roca & Raura Panaca Aillu \\
\hline Lloque Yupanqui & Avayni Panaca Aillu \\
\hline Maita Cápac & Usca Maita Panaca Aillu \\
\hline Cápac Yupanqui & Apu Maita Panaca Aillu \\
\hline Inca Roca & Vicaquirau Panaca Aillu \\
\hline Yahuar Huaca & Aucaylli Panaca \\
\hline Huiracocha & Socso Panaca Aillu \\
\hline Pachacútec & Hatun Aillu / Iñaca Panaca Aillu \\
\hline Túpac Yupanqui & Cápac Aillu \\
\hline Huaina Cápac & Tumipampa Aillu \\
\hline
\end{tabular}

3 Sapan Inca es el título del gobernante cuzqueño.

4 Aún cuando no cuestiona la oposición hanan-rurin, Rodolfo Cerrón-Palomino señala, sobre la voz «hurin», que, en verdad, este término nunca existió ni en quechua ni en aimara. La forma tendría su origen en una lectura equivocada de «lurin», como lo prueba la misma toponimia, no solo en la zona quechua, sino también en la aimara, conjuntamente con el hecho de que la voz «hurin», como tal, no se encuentra en los diccionarios coloniales. De esta forma, advierte que ésta sería una suerte de espejismo léxico desarrollado en oposición a «hanan». Así, aquí se opta por escribir «rurin» dada la aceptación generalizada del término y que «h» no estuvo asociada con esta voz. Véase Cerrón-Palomino, 2002. 
Esta relación de panacas es aceptada casi sin mayor cuestionamiento pese a haber ciertas discrepancias en torno a la existencia de la última panaca así como a la integración - $\mathrm{O}$ no- de los dos supuestos grupos asociados con Pachacútec: Iñaca Panaca y Hatun Aillu.

\section{UNA BREVE HISTORIA DE LA VOZ PANACA}

Es bastante común encontrar en los textos referentes a los incas menciones a las panacas e incluso la identificación de cualquier miembro de la nobleza incaica, prehispánica o colonial, como integrante de alguna de ellas. Los investigadores contemporáneos no sienten tampoco la necesidad de justificar estas asociaciones y por tanto la relación de panacas y la necesaria pertenencia de los nobles del Cuzco a alguna de ellas es asumida como una verdad historiográfica. Incluso en los textos escolares peruanos, a los que normalmente se trasladan las novedades historiográficas con cierto retraso, se encuentran explicaciones y referencias a las mismas. En esta oportunidad interesa empezar a escudriñar la historia de esta categoría. Por eso son importantes algunas preguntas iniciales: ¿̇cuándo se empezó a identificar a la nobleza cuzqueña como una organización dividida en panacas? ¿por qué utilizar la voz panaca para estas asociaciones?; es decir: ¿̇uál es la historia de esta categoría? Intentando responder a esta pregunta no es muy difícil llegar a una evidente conclusión: las fuentes coloniales no identifican a los incas divididos en nada parecido a panacas. Así por ejemplo, el Inca Garcilaso de la Vega, con evidente filiación a la nobleza cuzqueña, y con la firme determinación de mostrar este origen, jamás se identificó como miembro de alguna panaca. Por su parte, como es evidente, es Pedro Sarmiento de Gamboa la fuente principal de todas las asociaciones de la nobleza cuzqueña y su división en panacas. Sin embargo, al hacer la relación de nobles cuzqueños en la época de la redacción de su crónica a modo de justificación de sus afirmaciones, no los divide en panacas, aunque sí identifica a los nobles cuzqueños como miembros de aillus asociados con Incas reinantes. Seguramente algunos lectores estarán pensando en este momento en que existe una probanza colonial en la que algunos nobles cuzqueños se reclaman descendientes de Cápac Aillu, la panaca de Túpac Yupanqui (Rowe,1985). Sin embargo, como veremos, literalmente solo están manifestando que forman parte del aillu noble, el aillu de los cápac, de los ricos, los poderosos. Llama en ese sentido poderosamente la atención que este aillu, por alguna razón identificado posteriormente como una «panaca», no tenga ninguna referencia a ésta en su denominación. ¿De dónde viene entonces esta asociación? Empecemos por rastrear algunas de las funciones y roles que tienen las panacas según la historiografía.

Tal vez la definición más utilizada, aunque no por cierto totalmente aceptada, es la que plantea Tom Zuidema, quien las define como: «el grupo o unidad de hermanos con sus hermanas descendientes de un antepasado masculino en una línea masculina de hombre y línea femenina de mujeres por cuatro generaciones» (Zuidema, 1980: 77)5. Adicionalmente, Zuidema identifica roles calendáricos para las panacas y las asocia tanto con el sistema de ceques como con la irrigación del Cuzco, además de con los aillus cuzqueños (Zuidema, 1995 [1964]; 1980; 2004). Asimismo, establece una relación entre ellas y las tierras cuzqueñas a través de la identificación de cada panaca con un sistema de chapas (espacios agrícolas) a partir de una referencia de Juan de Betanzos. Por

5 Tom Zuidema ha trabajado numerosas veces sobre las panacas incaicas desde la redacción de su tesis doctoral. Con el paso del tiempo, los aportes de Zuidema en ese sentido son numerosos puesto que las ha estudiado desde diversas perspectivas teóricas. Sin embargo, la definición citada anteriormente, se ha mantenido a través del tiempo en sus investigaciones. 
su parte, María Rostworowski identificó una fuerte presencia femenina en las llamadas panacas a partir de la presencia de la raíz pana (hermana) por lo que asumió una filiación matrilineal para estos grupos (Rostworowski, 1983: 141 y ss.). Adicionalmente, esta autora identifica también tierras asociadas con las panacas incaicas tanto en el Cuzco como fuera de él. La bibliografía sobre los incas identifica además sistemáticamente dos funciones para estos grupos: cuidar la momia de su fundador, garantía de la existencia del grupo, y cuidar e incrementar la memoria del mismo (Bravo, 1986; Pease, 1992; Regalado, 1996a; Rostworowski, 1983; Zuidema, 1980; 2004). Se ha afirmado también que estos grupos eran en parte responsables de mantener las alianzas hechas por su fundador por lo que se les asocia con una evidente capacidad de convocatoria de mano de obra en los grupos étnicos, la misma que explicaría su poder y prestigio en el Tahuantinsuyo, expresado, por ejemplo, durante el proceso sucesorio (Hernández Astete, 2004).

Por su parte, la existencia simultánea de panacas y aillus en el Cuzco ha sido explicada a la luz de la organización tripartita de la ciudad planteada por Zuidema. Así, el grupo inca al que pertenecen las panacas se consideraría Collana y tendría más jerarquía que el grupo Cayao asociado tanto con los aillus que acompañaron a los Ayar en la fundación del Cuzco como con las poblaciones originarias de la región. Paralelamente, dada la exogamia del grupo inca, de sus uniones con mujeres Cayao surgió el grupo Payan, de mayor jerarquía que los de Cayao al ser descendientes de los Incas (Bravo, 1986; Zuidema, 1995 [1964]; 1980). Dentro de esta división, Collana se asociaría con los incas fundadores de panacas, Payan con las panacas mismas y Cayao con los aillus y las otras poblaciones cuzqueñas. De ese modo, dentro de esta postura, claramente las panacas se diferenciarían en rango de los llamados aillus. Sin embargo, Franklin Pease (1992: 73) puntualizó que tanto la supuesta descendencia de Huaina Cápac (Tumipampa Aillu), como la de Túpac Inca Yupanqui (Cápac Aillu), no incluían la voz panaca en su denominación, situación que complica la propuesta de diferenciar aillus de panacas, sobre todo cuando se asumía que los incas solo fundaban panacas. Situación similar se puede observar en el caso de Hatun Aillu, vinculado a Pachacútec, pues si bien Sarmiento menciona que éste se fusionó con Iñaca Panaca luego que Pachacútec se hiciera con la borla, María Rostworowski documentó la vigencia de ambas en la sociedad colonial (Rostworowski, 1983: 143)6. Adicionalmente, John Rowe (2003: 45 y ss.) llamó la atención acerca de los nombres de algunas panacas, tales como Hatun Aillu (aillu grande) y Cápac Aillu (aillu real) y menciona que éstas eran frases descriptivas y no nombres de panacas, situación que, unida al hecho que algunos aillus, como indica Rostworowski (1983: 150 y ss.), tenían ceques a su cargo y algunas panacas disponían únicamente de adoratorios, hizo pensar a Rowe que aillu y panaca fueron probablemente sinónimos antes del siglo XVI y que la caracterización de una panaca como el grupo de parentesco fundado por un Inca «reinante» sería más bien una construcción posterior a la Conquista. Adicionalmente, dada la confusión entre aillu y panaca, es necesario profundizar en la idea planteada por Rostworowski acerca del significado de la voz panaca (Rostworowski, 1983: 139) y estudiar detenidamente los nombres de las mismas, pues es probable también que la voz panaca aludiera únicamente a la descendencia femenina de los incas reinantes y no a toda la parentela del gobernante.

6 Para esta autora, Iñaca Panaca era una de las panacas añadidas. Asimismo, la considera de estructura matrilineal y dadora de mujeres. Es necesario aquí mencionar que el tema de la doble descendencia parece una incorporación colonial y el hecho de que algunas coyas provengan de Iñaca, parece explicarse por la tremenda conveniencia de los aspirantes a Incas de relacionarse con la descendencia de Pachacútec. Véase un análisis del tema en Hernández Astete, 2002. 
En ese sentido, como se ha mencionado, está tan enraizada en la historiografía incaica la identificación de los grupos de poder al interior de la nobleza cuzqueña con la voz panaca que nadie considera necesario citar ninguna referencia al hablar de ellas e identificar a miembros de la nobleza cuzqueña como militantes de las mismas. Sin embargo, todos los textos parecen tener un origen común: la obra de Luis E. Valcárcel, quien en 1925 presenta, por primera vez, la división de la élite incaica en 10 panacas. Afirmaba Valcárcel que fue Bartolomé de las Casas quien, en su Historia Apologética de las Indias, presenta la información sobre estos grupos al relacionar a Pachacútec con la organización del Cuzco, en realidad de la élite incaica, en dos grupos: Alto y Bajo Cuzco. En la versión de esta reorganización que presenta Las Casas, y resaltaba Valcárcel, la nobleza habitante de ciudad sagrada fue dividida, en realidad subdivida, en 10 panacas que Pachacútec asignó a sus antecesores e inmediato sucesor (Valcárcel, 1925: 35-37):
1. Chima
2. Raurahua
3. Hahuainñin
4. Uscamaita
5. Apumaita
6. Huekaquirau
7. Aucailli
8. Sucsu
9. Iñaca
10. Atum

Posteriormente, en el mismo texto, Valcárcel define Pana-kak como: «... la que es hermana» y añade:

La «panaca» es, pues, la fratría, la agrupación fraterna [...] Los Ayllus cuzqueños, fundadores del imperio, guardaron relativamente buena armonía, por eso se consideraron hermanos, y la fundación de las panacas correspondió a una necesidad de índole política: el parentesco de todos (Valcárcel, 1925: 38).

Aunque, como veremos, la interpretación que Valcárcel hace del término no es del todo equivocada, ignoramos las razones por las que concluye a partir de la referencia de Las Casas en la identificación de las «panacas» con el concepto que maneja de fratría y que, a nuestro entender, constituye el texto fundador de la interpretación y el significado que hoy se le da al término. De hecho, para este autor, la formación de las «panacas» y su identificación con las fratrías, constituye parte de la «evolución natural» de cualquier sociedad. La información de Valcárcel, en la que la nobleza incaica se divide en panacas, fue paulatinamente incorporada por la historiografía de manera sistemática sin hacerse necesaria su justificación. De ese modo, como se viene afirmando, los textos incorporaron esta división de la élite incaica en grupos más pequeños y los etiquetó como «panacas» sin que exista ninguna evidencia clara que la sostenga.

Encontramos también en el texto de Valcárcel, en claro reconocimiento de la autoridad de Las Casas sobre el tema, una clara oposición a la visión de Sarmiento pues considera que dichos grupos no fueron formados históricamente sino que integraban un sistema que Pachacútec sólo reorganizó. Asimismo, identifica a Cápac Aillu como una suerte de «panaca principal», pues asume el rol de «panaca reinante», el mismo que perdería luego de la sucesión; tema sobre el que volveremos más adelante (Valcárcel, 1925: 90 y ss.). 


\section{LAS PANACAS Y LAS FUENTES COLONIALES}

Lo mencionado hasta ahora nos obliga a revisar las fuentes disponibles en búsqueda de la información que aparentemente sostiene la definición de panaca y la que nos permita aclarar su significación, pues, como se ha mencionado, sin temor a equivocarnos podemos afirmar que no existen referencias en la documentación colonial sobre la división de la élite en «panacas» y ningún noble cuzqueño declara haber pertenecido a alguna de ellas. Debo mencionar, sin embargo, que esto no significa que no existieran grupos dentro de la nobleza cuzqueña asociados con los Incas reinantes. Simplemente que, al parecer, no hay ningún argumento válido para identificarlos como «panacas» y que la estructura organizativa del Cuzco — sobre la que se sustenta su autoridad - parece haber sido distinta a la que la historiografía asume hasta el momento, como veremos.

Aun cuando está totalmente extendida la información que presenta Pedro Sarmiento de Gamboa sobre la formación histórica de las panacas, existe un grupo de textos, liderados por el Padre Las Casas, en los que se manifiesta un sistema distinto.

En relación a su supuesto origen histórico, aparentemente, ante un pedido de Sarmiento de Gamboa al virrey Francisco de Toledo hecho en Cuzco el 29 de febrero de 1572 para autentificar su manuscrito, se mandó llamar a los más nobles de la ciudad a través de Gabriel de Loarte, de lo que da fe Álvaro Ruiz de Navamuel. Es importante notar que en la relación de a quiénes se les leyó la obra de Sarmiento para que testifiquen la autoridad de las informaciones, en una suerte de construcción de la autoridad del manuscrito, se les relaciona con los aillus de los doce Incas, y que jamás se menciona nada parecido a las doce panacas (Sarmiento, 1988 [1572]: 172 y ss.).

Por otra parte, en la obra de Sarmiento, a diferencia del sistema planteado por Bartolomé de las Casas, se asume la creación de los linajes del Cuzco de manera sucesiva:

Este Manco Capac ordenó, para conservación de su memoria, lo siguiente: que su hijo mayor y de su mujer legítima, que era su hermana, sucediese en el estado, y si hubiese hijo segundo, a éste tiene cargo que tuviese cuidado de amparar a todos los demás hijos y parientes, y que ellos le reconociesen por cabeza para sus necesidades, y que se apellidasen de su nombre, y él tuviese cargo de los favorecer y sustentar, y para esto les dejó hacienda. A esta parcialidad o bando o linaje llamó ayllu, que es lo mismo que linaje. Y si faltase hijo segundo, y aunque hubiese, si era incapaz de gobierno, lo encargasen al pariente más cercano y de más habilidad. Y para que de él tomasen los venideros ejemplo, hizo el primer ayllu y llamole Chima panaca Ayllo, que quiere decir linaje que desciende de Chima; porque el primero a quien dejó encomendado su linaje o ayllo se llamó Chima, y Panaca quiere decir descender (Sarmiento, 1988: 63-64).

Sarmiento da cuenta de la formación de cada uno de los once grupos en los que divide a la nobleza cuzqueña, sin llamarlos nunca como «panacas». Al respecto de estos grupos, reproducimos la relación de los mismos según la obra de Pedro Sarmiento de Gamboa. En el cuadro se aprecia tanto el nombre del inca fundador como el de la parcialidad a la que pertenecía cada uno (Hanan Cuzco o Rurin Cuzco) además del nombre del «ídolo huauqui» de cada inca. 


\begin{tabular}{|c|c|c|c|c|}
\hline & Parcialidad & Fundador & Panaca & Huauqui \\
\hline 1 & Rurin Cuzco & Manco Cápac & Chima Panaca Aillu & Pájaro indi \\
\hline 2 & Rurin Cuzco & Sinchi Roca & Raura Panaca Aillu & Huanachiri / Amaru (pez) \\
\hline 3 & Rurin Cuzco & Lloque Yupanqui & Avayni Panaca Aillu & Apu Maita \\
\hline 4 & Rurin Cuzco & Maita Cápac & Usca Maita Panaca Aillu & Pájaro indi? \\
\hline 5 & Rurin Cuzco & Cápac Yupanqui & Apu Maita Panaca Aillu & ¿? \\
\hline 6 & Hanan Cuzco & Inca Roca & Vicaquirau Panaca Aillu & ¿? \\
\hline 7 & Hanan Cuzco & Yahuar Huaca & Aucaylli Panaca & Inca Amaru \\
\hline 8 & Hanan Cuzco & Huiracocha & Socso Panaca Aillu & Chuqui-Ylla / Inti illapa \\
\hline 9 & Hanan Cuzco & Pachacútec & Hatun Aillu / Iñaca Panaca Aillu & Cusi-Churi \\
\hline 10 & Hanan Cuzco & Túpac Yupanqui & Cápac Aillu & Huaraqui Inca \\
\hline 11 & Hanan Cuzco & Huaina Cápac & Tumipampa Aillu & \\
\hline
\end{tabular}

De acuerdo a la información de Sarmiento de Gamboa, existieron en el Cuzco once grupos (panacas), cinco de Rurin Cuzco y seis de Hanan Cuzco. Sin embargo, para Cristóbal de Molina, que terminó de escribir su obra hacia 1575 (Molina, 1988 [1575?]), la nobleza cuzqueña estuvo dividida en diez linajes según la información que se desprende del ritual que presenta al describir la fiesta de la Citua en el que participan los grupos que Molina asocia con cada una de las cuatro partes que formaban el Tahuantinsuyo:

\begin{tabular}{|c|c|c|c|}
\hline \multicolumn{2}{|c|}{ Rurin Cuzco } & \multicolumn{2}{c|}{ Hanan Cuzco } \\
\hline Contisuyo & Collasuyo & Antisuyo & Chinchaisuyo \\
\hline Chima Panaca Aillu (1) & Ahuaymin Aillu (3) & Aucailli Aillu (7) & Vicaquirao Aillu (6) \\
Yaura Panaca Aillu (2) & Uscamaita Aillu (4) & Cuzco Panaca Aillu (8) & Atun Aillu (9) \\
& Apomayta Aillu (5) & & Cápac Aillu (10) \\
\hline
\end{tabular}

El hecho de que Molina no mencione a Tumipampa Aillu, la supuesta panaca de Huaina Cápac, parece corresponder, desde una perspectiva histórica de su formación, al momento recordado por sus informantes, aparentemente asociado a un tiempo anterior al gobierno de Huaina Cápac. Sin embargo, la asociación que hace de las mismas con cada uno de los cuatro suyos que formaban el Tahuantinsuyo nos da una pista sobre la organización cuzqueña, pues se sabe que esta distribución estaba relacionada con la existencia de una suerte de geografía sagrada andina, la misma que estudió Tom Zuidema7 (1995 [1964]) en su trabajo sobre los ceques o líneas imaginarias que partían del Cuzco y en las que se distribuían 328 huacas. De acuerdo a la hipótesis de Zuidema, tanto los ceques como los adoratorios se asociaban con la organización cuzqueña.

Según la bibliografía sobre el tema, básicamente sustentada en la información de Sarmiento, las «panacas» tomaban el nombre de su primer jefe según una tradición iniciada, en opinión de Sarmiento, por Manco Cápac, cuyos descendientes integraron Chima Panaca Aillu, liderada inicialmente por Chima, pariente de Manco Cápac (Sarmiento, 1988: 63-64) y primer responsable del grupo. Esta situación se repite en la mayoría de los casos salvo

7 Sobre el espacio sagrado que significó el Tahuantinsuyo, véase también Regalado, 1996b. 
en el de los descendientes de Pachacútec, que según el mismo cronista, debido a la gran cantidad de hijos del Inca (cuatro en su mujer principal y cien varones y cincuenta mujeres en sus esposas secundarias) llamó Hatun Aillu, que quiere decir «gran linaje» (Sarmiento, 1988: 126). En este caso, habría que preguntarse por el tipo de pertenencia de todos estos hijos al grupo pues, si la panaca estaba integrada simultáneamente por los hijos de la coya y los de las esposas secundarias del Inca, no tendrían todos sus integrantes las mismas prerrogativas al interior del grupo. En ese sentido, se cree por ejemplo que solo los hijos de la coya podían convertirse en Incas ${ }^{8}$, situación sobre la que volveremos más adelante.

Adicionalmente a la existencia de estos linajes cuzqueños, las crónicas mencionan aillus conformados por pobladores del Cuzco, como los diez aillus que según Sarmiento de Gamboa acompañaron y ayudaron a los incas en la fundación del Cuzco dentro del ciclo mítico de los hermanos Ayar. En este mismo contexto, Sarmiento menciona también a algunos descendientes de estos aillus cuzqueños que vivían en la zona en 1572, fecha en que termina de redactar su obra (Sarmiento, 1988: 52-53):

\begin{tabular}{|l|l|}
\hline \multicolumn{1}{|c|}{ Hanan Cuzco } & \multicolumn{1}{|c|}{ Rurin Cuzco } \\
\hline 1. Chauin Cuzco Aillu (descendientes de Ayar Cachi) & 6. Sutic-tocco Aillu (generación que salió de la \\
2. Arayraca Aillu Cuzco-Callan (descendientes de & ventana del mismo nombre) \\
Ayar Uchu) & 7. Maras Aillu (generación que salió de Maras-tocco) \\
3. Tarpuntay Aillu & 8. Cuycusa Aillu \\
4. Huacaytaqui Aillu & 9. Masca Aillu \\
5. Sañoc Aillu & 10. Oro Aillu \\
\hline
\end{tabular}

Sobre la participación de la élite en el ejercicio del poder incaico, la historiografía ha centrado su atención, casi exclusivamente ${ }^{9}$, en su influencia en la sucesión incaica, dejando de lado la imagen de que en todos los otros contextos era el Inca quien decidía sobre todos los aspectos asociados con el gobierno. Sin embargo, todo parece indicar que, como manifiesta Regalado (1996a), la presencia e influencia de la élite en la organización del Tahuantinsuyo fue constante y que el Sapan Inca debía necesariamente negociar con ella dado que los linajes incaicos (panacas) participaban activamente en las decisiones y labores políticas al interior de la organización incaica (Hernández Astete, 2004).

Según la bibliografía existente, las panacas incaicas dependían casi exclusivamente de dos situaciones claramente identificadas: la existencia y cuidado del cuerpo de su fundador, que sustentaba su origen y permanencia en la élite, y el prestigio adjudicado a su ancestro, que afianzaba su poder. Evidentemente, la posición de una panaca dentro de la estructura de la élite, dependía también de su capacidad para negociar con los distintos sectores de la misma.

De este modo, siguiendo a Sarmiento, se asume que la organización cuzqueña fue construida históricamente y que las panacas fueron formadas cada vez que un nuevo Inca era entronizado. Sin embargo, como se ha mencionado, las fuentes sugieren también la existencia de un sistema alternativo. Ese es el caso que Valcárcel destacó en 1925 a partir de la obra de Bartolomé de las Casas. Así, según este cronista, Pachacútec dividió la ciudad sagrada en dos barrios, partes o bandos: Hanan Cuzco y Rurin Cuzco y luego hace una subdivisión en cada una de estas parcialidades, identificando cinco sectores en cada una de las parcialidades. Conviene citarlo en extenso:

8 Véase sobre el tema Regalado, 1996a; Rostworowski, 1983.

9 Para una postura distinta sobre la participación de la élite en el ejercicio del poder, véase Regalado, 1987 y $1996 a$. 
Lo primero que cerca de esto hizo, fué dividir toda la ciudad del Cuzco, que ya era muy populosa, en dos barrios o partes o bandos. El uno y más principal llamó Hanancuzquo, que quiere decir «la parte o barrio o bando de arriba del Cuzco»; a la otra puso nombre Rurincuzquo, que significa, «la parte o barrio de abajo del Cuzco». El barrio y parte Huanancuzquo, que era el principal, subdividió en cinco barrios o partes: al uno y principal nombró Capac ayllo, que quiere decir «el linaje del Rey»; con éste juntó gran multitud de gente y parte de la ciudad, que fuesen de aquel bando; al segundo llamó Iñaca panaca; el tercero Cucco panaca; el cuarto Aucayllipanaca, el quinto Vicaquirau panaca; a cada uno de los cuales señaló su numero grande de gente, y así repartió por bandos toda la ciudad. Del primer barrio o bando hizo capitán a su hijo mayor y que le había de suceder en el reino; el segundo y tercero señaló a su padre y descendientes por la línea trasversal; el cuarto a su abuelo y descendientes también por la línea transversal; el quinto a su visabuelo, por la misma línea. Asimismo la parte y bando segundo y principal de la ciudad que llamó de Rurincuzco, barrio de abajo del Cuzco, subdividió en otras cinco partes o parcialidades: a la primera llamó Uzcamayta, y de éste hizo capitanes a los descendiente del segundo hijo del primer Rey Inga; a la segunda nombro Apomaytha, de la cual constituyó capitán y capitanes al segundo hijo y descendientes del segundo Inga; a la tercera parcialidad o bando puso nombre Haguayni, del cual nombró por capitán y capitanes al segundo y descendientes del tercero Inga; al cuarto barrio nombró Rauraupanaca, cuya capitanía encomendó al segundo hijo y descendientes del cuarto Inga; al quinto barrio llamó Chimapanaca, y dióle por capitán y capitanes al segundo hijo y sus descendientes del quinto Inga (Las Casas, 1948: 90-91).

Las Casas menciona también que Pachacútec señaló capitanes para cada uno de estos «barrios» y los asignó a sus ancestros y sus respectivos descendientes así como a los descendientes de los Incas anteriores y sus respectivos familiares. Evidentemente cada uno de los barrios, que hoy llamaríamos panacas, asociados con determinados Incas o sus descendientes, estarían integrando también a todos los familiares del fundador, «descendientes en línea transversal» según el citado cronista. Así, en una organización presidida por el Sapan Inca, en Hanan Cuzco, Pachacútec habría entregado el primer barrio a su hijo, el segundo y tercer barrio a su padre, el cuarto a su abuelo y el quinto a su bisabuelo, respectivamente. En el caso de Rurin Cuzco, la situación habría sido otra en opinión de Las Casas, pues cada barrio estaba encargado a los segundos hijos de los Incas anteriores, desde el primero al cuarto. Es curioso el sistema que propone Las Casas y que se reproduce en el siguiente cuadro, pues presenta una clara diferenciación entre el sistema de reparto del poder entre cada una de las parcialidades del Cuzco, situación que puede explicarnos un tema aún no resuelto en la historiografía incaica, el de la sucesión, dadas las constantes contradicciones sobre el sistema sucesorio incaico que aparecen en las fuentes10. A continuación se presenta un cuadro que resume la información que proporciona Las Casas, el mismo que se completa con los nombres de los incas, según la relación tradicional de reyes, con los que estaría relacionado cada grupo y que correspondería a su ancestro fundador.

10 Aún cuando la temática de este artículo no permite extendernos sobre el tema sucesorio, se debe mencionar que pese a que exista cierto consenso acerca de la «habilidad« como criterio predominante en la sucesión, las fuentes proporcionan imágenes aparentemente contradictorias sobre el modo de acceso al poder, las mismas que pueden resolverse desde una perspectiva que asuma la sucesión de modo paralelo pues es claro que existieron dos sectores diferenciados en la élite incaica Hanan y Rurin y que, pese a manejarse al interior de la hipotesis de la dualidad, quienes estudian la sucesión no la han resuelto desde este mismo marco teórico. Sobre el tema de la sucesión, ver por ejemplo: Regalado, 1996a y Rostworowski, 1983. 


\begin{tabular}{|l|l|l|l|}
\hline \multicolumn{1}{|c|}{ Hanan Cuzco } & \multicolumn{1}{|c|}{ Encargado/Ancestro } & Rurin Cuzco & \multicolumn{1}{c|}{ Encargado/Asociado } \\
\hline 1. Cápac Aillu & Su hijo (Túpac Yupanqui) & 1. Usca Mayta & $\begin{array}{l}\text { El segundo hijo del primer Inca } \\
\text { (Manco Cápac) }\end{array}$ \\
\hline 2. Iñaca Panaca & Su padre (Huiracocha) & 2. Apomayta & $\begin{array}{l}\text { El segundo hijo del segundo Inca } \\
\text { (Sinchi Roca) }\end{array}$ \\
\hline 3. Cucco Panaca & Su padre (Huiracocha) & 3. Huaguani & $\begin{array}{l}\text { El segundo hijo del tercer Inca } \\
\text { (Lloque Yupanqui) }\end{array}$ \\
\hline 4. Aucaylli Panaca & Su abuelo (Yahuar Huaca) & 4. Raura Panaca & $\begin{array}{l}\text { El segundo hijo del cuarto Inca } \\
\text { (Maita Cápac) }\end{array}$ \\
\hline 5. Vicaquirau Panaca & Su bisabuelo (Inca Roca) & 5. Chima Panaca & $\begin{array}{l}\text { El segundo hijo del quinto Inca } \\
\text { (Cápac Yupanqui) }\end{array}$ \\
\hline
\end{tabular}

Es preciso detenerse en dos detalles claves según el sistema que presenta Las Casas. Por un lado, el hecho de que esta relación aparentemente sería reorganizada ante la presencia de un proceso sucesorio, en el que el nuevo Inca reordenaría todo el sistema a partir del parentesco con él. Asimismo, conviene mencionar algo que desarrollaremos más adelante y es la identificación de Cápac Aillu con Túpac Llupanqui pues si seguimos el sistema planteado por Las Casas, él estaría encargado de este grupo únicamente mientras sea asociado como hijo del Inca y que, apenas otro personaje asumiera el mando, Cápac Aillu debería ser dirigido por el hijo del nuevo Inca reinante. Al respecto, recordemos que Valcárcel identificaba a este grupo como una suerte de «panaca reinante» o aillu principal. A nuestro entender sin embargo, y como explicaremos más adelante, Cápac Aillu sería el nombre genérico de la nobleza incaica, el mismo que estaría integrado por grupos asociados con cada uno de los Incas convertidos en ancestros. Esta sería la razón por la que las probanzas coloniales presentadas por nobles incaicos, como la mencionada Probanza de los Incas nietos de conquistadores, como un medio de demostrar su origen noble, afirmaran pertenecer a este grupo. Asimismo, la razón por la que la historiografía identifica a Cápac Aillu con el linaje de Túpac Yupanqui, correspondería únicamente al momento recordado por los nobles incaicos, la división de la ciudad por parte de Pachacútec, donde el jefe del grupo era Túpac Yupanqui. Esta situación, unida a la evidente historización de los incas realizada por Sarmiento, explicaría la división en panacas y la identificación de Cápac Aillu exclusivamente con Túpac Yupanqui, quien a nuestro entender, dejó de ser el «capitán de este aillu» en el momento en que se ciñó la borla.

Información similar a la que presenta Las Casas es la que nos ofrece Gutiérrez de Santa Clara, quien afirma que Túpac Yupanqui:

... fue el primer Inga que comenzó hacer la fortaleza del Cuzco, aunque otros viejos dicen que Pachacuti Capac Inga la comenzó edificar cuando tuvo las competencias con el gran curaca del Cuzco, y que no la pudiendo acabar la dexó y que después este Topa Inga Yupangue la añadió mucha parte, desde donde dava mucha guerra al gran curaca. Asimismo dividió la ciudad en dos barrios grandes: el uno y el más principal llamó Annan Cuzco, que quiere decir el barrio de arriba del Cuzco y el otro se llamó Hurin Cuzco, que significa el barrio de abaxo. Hecho esto repartió el barrio de arriba en cinco partes: al primero y más principal llamó Ayllo cappa, que quiere decir el barrio del linaje del Inga; el segundo se llamó Yña Capañaca [Iñaca panaca], la tercera Cuccopanaca [Sucso panaca]; la cuarta Yllipanaca [Aucalli panaca], y la quinta Cumapanaca [Suma panaca]; y a cada uno destos barrios señaló número de gente para que, como vecinos perpetuos, estuviesen en el. Del primer barrio hizo capitán y señor a un hijo que tenía que le había de suceder en el reino; el segundo y el tercero señaló para sus descendientes por línea transversal; el cuarto a su abuelo y 
decendientes; el quinto a su bisabuelo. El otro barrio segundo, que era la otra parte de la ciudad de abaxo, repartió en otras cinco partes: a la primera llamó Uzcamayta [Usca Mayta] y desta hizo capitán a los descendientes del segundo hijo del primer Inga que reinase después dél; la segunda nombró Appomayta [Apo Mayta], de la cual constituyó por capitán al hijo segundo del segundo Inga. Y por esta orden mandó que en el tercero, cuarto y quinto barrio sucediesen en la administración los segundos hijos del tercero, cuarto y quinto Inga, aunque después no creció este imperio por la entrada que hicieron los españoles en la tierra (Gutiérrez de Santa Clara, III: 214, citado por Zuidema, 1995 [1964]: 224).

Por su parte, Fray Martín de Murúa, en un capítulo sin numerar ubicado entre el segundo y el tercer capítulo según la edición preparada por Manuel Ballesteros en 2001 a partir del conocido «Manuscrito Wellington» (2001), titulado «Del primer rey ynga Mango Capac, padre y genitor, de quien procede y se derivan todos los demás y de sus maravillosos hechos», presentaría también una información similar, la que citamos también en extenso para poder identificar una tendencia distinta a la presentada por Sarmiento y asumida por la historiografía. En ese sentido, refiriéndose también a Pachacútec, afirmaba el mercedario que:

Lo primero que hizo fue reducir a policía la dicha ciudad, dando forma de pueblo a lo que antes era chacarras o caseríos, partiéndola en dos ayllos o barrios, como se dirá en la discreptión de la dicha ciudad del Cuzco, con que autorizó su persona y se hizo respetar más de la gente popular. Nombró por capitán a su hijo Sinchiroca de uno de los ayllos en que repartió, que después de sus días le había de suceder en la corona. Los demás los repartió entre sus descendientes por línea transversal, haciendo ley que los hijos segundos de los Yngas viviendo sus padres gobernasen los ayllos yparcialidades, como cabezas y capitanes generales (Murúa, 2001: 42-43).

Como es evidente, son dos las explicaciones que ofrecen las fuentes coloniales acerca de la división de la élite incaica. Por un lado la formación de grupos de manera histórica, y de otro, aquella que presenta un sistema cambiante en la que todo se reordena según el parentesco con el inca reinante. Queda claro además que en ningún caso se encuentra en la documentación colonial ninguna referencia a que estos grupos deban etiquetarse como panacas. Evidentemente, al parecer, tanto Las Casas como Gutiérrez de Santa Clara y Fray Martín de Murúa manejaron una fuente común, quizás la crónica aún hoy extraviada de Cristóbal de Molina. De hecho, aunque Las Casas nunca estuvo en el Perú, se sabe de su vinculación con textos de la época y de su preocupación por documentarse sobre el área andina. El solo hecho que escriba Rurin Cuzco, en vez de Urin Cuzco, de la misma forma que Molina, parece indicar que tuvo acceso a fuentes confiables en cuanto a la manera de escribir quechua 11.

\section{EN BÚSQUEDA DE UNA ETIMOLOGÍA DE PANACA}

Varias son las explicaciones que la historiografía ha otorgado a la voz panaca. Conviene recopilar las que se han mencionado hasta el momento y compararlas con las que se encuentran en los textos coloniales a fin de buscar una explicación al término. Así, Zuidema

11 Aún cuando las ediciones de Molina consignen Hurin Cuzco, la consulta directa con el manuscrito no deja ninguna duda sobre el hecho de que esto se debe a una mala lectura de Rurin, claramente en el original ubicado en la Biblioteca Nacional de Madrid. Para una explicación sobre la naturaleza de Hurin Cuzco y su identificación como un espejismo léxico, véase Cerrón-Palomino, 2002. 
las define como: «el grupo o unidad de hermanos con sus hermanas descendientes de un antepasado masculino en una línea masculina de hombre y línea femenina de mujeres por cuatro generaciones» (Zuidema, 1995 [1964]; 1980: 77). Por su parte, María Rostworowski, asumiendo una identificación «matrilineal» para estos grupos, asume con Zuidema que, dadas las características femeninas del término pana (hermana), cada hombre pertenecería al grupo de su hermana por lo que sus hijos no podrían clasificarse con él, aunque esta autora no resuelve cuestiones centrales acerca de la manera en que cada uno de los nobles cuzqueños se identificaba con estos grupos (Rostworowski, 1993: 138 y ss.). Adicionalmente, como se mencionó, Valcárcel afirmaba que Pana-kak podría leerse como «... la que es hermana...» sin explicar la presencia de varones al interior de estos grupos (Valcárcel, 1925: 38).

Ahora bien, el escudriñamiento de este término en las fuentes coloniales nos otorga mayores precisiones sobre el significado de panaca. Para Sarmiento de Gamboa, panaca significaba «descender» por lo que entiende frases como chima panaca aillo como «el linaje que desciende de Chima», el primer responsable del grupo en su versión de la formación histórica de las panacas (Sarmiento, 1988: 63-64).

Como es sabido, hay en quechua dos términos que corresponden al significado de «hermana»: pana y ñaña. El varón llama pana a su hermana, mientras que la mujer le dirá ñaña. Paralelamente, huauque y tora corresponden a «hermano», por lo que el varón llama huauque a su hermano, mientras que la mujer lo llama tora (González Holguín, 1975 [1607]). A su vez, dada la inexistencia de términos para identificar a los colaterales, estos mismos se aplicarán para nombrarlos. Según el propio González Holguín:

«... todos los primos hermanos, y segundos y terceros se llaman desta misma manera como hermanos y hermanas huauque, ñaña, pana, tora, con los nombres de hermanos, que no hay de primos» (González Holguín, 1989, citado por Cerrón-Palomino, 1993: 251).

Es por eso que el mismo González Holguín, esta vez en el Vocabulario, al señalar el significado de pana, lo extiende a los colaterales:

«pana: hermana del varón, o prima hermana, o segunda, o de su tierra, o linage o conocida» (González Holguín, 1975).

No se encuentra, sin embargo, en los diccionarios coloniales, ninguna definición de panaca como «linaje» o «grupo de parentesco», la que sí corresponde, sin ninguna duda, al término aillu. En ese sentido, Fray Domingo de Santo Tomás identifica tanto «linaje» como «familia» con la voz aillu (Domingo de Santo Tomás, 1951 [1560]).

En la búsqueda de una descripción etimológica para panaca, dado que ésta no es registrada en ningún diccionario colonial, para su reconstrucción se debe partir necesariamente de la raíz nominal pana que refiere a la «hermana» cuando el que habla es un varón. En este sentido, en la probable formación de panaca, la incompatibilidad de la raíz nominal pana con la marca de agentivo / $q$ / hace imposible la formación de pana(q), pues esta marca agentiva exige la presencia de un verbo. Así, la necesaria verbalización de la raíz nominal puede realizarse con el auxiliar /ka-/ («ser»)12. Con la presencia de este auxiliar se hace posible admitir el agentivo $q$ para formar la construcción /pana-ka-q/. A partir de esta forma, debido al uso frecuente de la expresión se produce el desgaste del auxiliar /-Ka/, llegando incluso a desaparecer, para dar pana-q; es esta forma la que recibe finalmente la vocal paragógica, obligatoria en el aimara, como resultado de la influencia de esta lengua. De 
otro lado, la presencia de la vocal /a/ final se puede explicar por la exigencia, en el aimara, de una vocal final en las palabras acabadas en consonante. Así llegamos a pana-ka-q(a), cuyo significado sería «las que son hermanas de...». De esta manera, la construcción quedaría finalmente como panaq(a), que por cierto nunca tendría el significado de «linaje» y, en consecuencia, como intuía Valcárcel, construcciones del tipo Chima Panaca Aillu, referirían a «las que son hermanas de Chima». Por su parte, frases como Iñaca Panaca, podrían vincularse o con una redundancia, dado el significado de «hermana» para ambos términos en quechua y aimara respectivamente, o responder a una cuestión estilística, explicada también por la cercanía entre el quechua y el aimara13.

Así, si entendemos que la voz panaca está asociada, únicamente, con las mujeres nobles, con las que son «pana» - pues se definen a partir del parentesco con alguno de los incas anteriores convertidos en ancestros-, se puede entender la filiación matrilineal de la más alta nobleza dentro de un sistema que asocia la poliginia incaica con el sistema matrilineal de parentesco. De este modo, solo integraban la nobleza cuzqueña más alta aquellos hombres y mujeres que descendían de una «pana», pues los hombres del grupo, incluyendo al Inca, tenían hijos también con mujeres que no formaban parte de la gran nobleza cuzqueña. Así, la pertenencia de un varón al «linaje real», a la nobleza, se explica por ser hijo de una «pana», mientras la condición noble de la mujer está asegurada por su caracterización como una «hermana de...». Solo las «pana» otorgaban el estatus de noble a sus descendientes directos puesto que, por lo que se sabe hasta el momento, la poligamia fue una prerrogativa de los varones. De este modo, un Inca, o cualquier descendiente del mismo, podía tener hijos con varias mujeres, pero solo los hijos de las mujeres nobles integraban la nobleza. Por su parte, todas las mujeres de la élite, hijas también de padres nobles, se definen como «panas» y constituían grupos de parentesco asociados con cada uno de los sectores del poder cuzqueño, y afirmaban su autoridad $-y$ su nobleza - por el parentesco con alguno de los Incas, a quienes rendían culto. Los aillus formados a partir de estos grupos de mujeres serían los que la historiografía ha denominado «panacas», salvo los casos de Cápac Aillu y Hatun Aillu que, como se ha mencionado, no tienen la voz panaca en su denominación.

\section{EN BÚSQUEDA DE UNA EXPLICACIÓN}

Si se asume la explicación anterior, como se ha señalado, quedan fuera de la caracterización como «panacas» tanto Cápac Aillu como Hatun Aillu. Empecemos por buscar una explicación para Cápac Aillu. Aquí vale la pena recordar el interés de Valcárcel en otorgar un mayor rango a Cápac Aillu, al punto de asociarlo con una suerte de realeza o panaca principal, protagonismo que Zuidema también ha señalado en varios de sus trabajos a este grupo, aunque sin dejar de clasificarlo dentro del sistema de 10 panacas (Valcárcel, 1925; Zuidema, 2004). Ahora bien, si atendemos exclusivamente al significado de Cápac Aillu, evidentemente debemos pensar en algo como: el aillu de los cápac, de los ricos, los poderosos. Con esta explicación, se puede asumir, sin temor a equivocarnos, que Cápac Aillu era únicamente el nombre genérico de la más alta nobleza incaica, la llamada realeza, por lo que cobra sentido entonces que, en las probanzas coloniales que se mencionó anteriormente, los nobles incaicos estén interesados únicamente en mostrar su filiación con Cápac Aillu; es decir, su condición de nobles. En este sentido entonces, podríamos deducir

13 La explicación de la reconstrucción de la voz panaca fue hecha por Rodolfo Cerrón-Palomino en una muy gentil comunicación personal en el 2005. 
que en la época de predominio incaico, el grupo de nobles era llamado Cápac Aillu, y que era de este aillu de donde salían los gobernantes y los distintos rangos de mayor autoridad cuzqueña. Asimismo, al interior de este grupo, existirían una serie de subgrupos integrados en principio por las hermanas de un Inca reinante y los descendientes de las mismas. Estos serían los diversos aillus caracterizados hasta ahora como panacas y cuya significación corresponde con los aillus de las hermanas de cada inca y sus descendientes. Como se ha mencionado, para ser noble, para integrar Cápac Aillu, era necesario descender de una de las «pana» y de un varón noble evidentemente.

Finalmente, queda por explicar el caso de Hatun Aillu que, como se mencionó líneas arriba, la historiografía asocia, no sin discrepancias, con Pachacútec, como una suerte de subdivisión de Iñaca Panaca. Es importante recurrir otra vez al significado literal del término: el de «Aillu grande». En este sentido, podríamos suponer una suerte de nobleza de menor rango que Cápac Aillu (quizás los Ilamados incas de privilegio) en la que se estaría integrando a todos los descendientes de nobles en mujeres no incas. La clave está en el texto del propio Pedro Sarmiento de Gamboa, quien al hablar de Pachacútec, menciona que éste:

Tuvo cuatro hijos legítimos en su mujer Mama Anahuarqui, tuvo cien hijos varones y cincuenta hijas bastardas, a los cuales, por ser muchos, Ilamó Hatun Ayllu, que quiere decir «gran linaje». Llámase por otro nombre este linaje Inaca Panaca Ayllu (Sarmiento, 1988: 126).

Así, pese a la necesidad de Sarmiento de construir linajes para los incas a modo de dinastías, deja también claro el origen de los integrantes de Hatun Aillu, el grupo de descendientes de los incas en mujeres no nobles, o de las élites locales, condición que debió tener por ejemplo Paullu Inca. Finalmente, y como una probable explicación a la última parte de la cita anterior donde queda clara la identificación de Hatun Aillu con Iñaca Panaca, dentro de los descendientes de los incas en mujeres de las diversas élites provinciales, hatun remite a los hombres e iñaca a las mujeres, aunque esto último queda pendiente de investigación.

Con esta explicación, la nobleza cuzqueña — cantera de la autoridad en el mundo prehispánico- estaría integrada por Cápac Aillu, que estaría comandado por el hijo del gobernante de turno —-según Las Casas-, e integrada por grupos de poder asociados por línea materna con los antiguos gobernantes — las llamadas panacas-. Asimismo, Hatun Aillu correspondería a los descendientes de los nobles en mujeres no incas, condición que recuerda el modelo tripartita de Zuidema en el que Collana identificaría a Cápac Aillu, Pallán a Hatun Aillu y, finalmente, Cayao a las élites locales, las que, para acceder al poder cuzqueño, establecían vínculos de parentesco con los incas, tema por cierto bastante documentado por la literatura que se refiere al Tahuantinsuyo. Corrobora esta hipótesis la evidencia léxica presente en el único documento colonial que se preocupa por sistematizar el parentesco incaico, el Vocabulario Anónimo de 1586, donde encontramos linage como aillu, linage real como «capac aillu» y linage noble como «collana hatun ayllu» (Anónimo, 1951: 155).

\section{Referencias citadas}

ANÓNIMO, [1586] 1951 - Arte y Vocabvlario en la lengva general del Perv, 221 p.; Lima: Antonio Ricardo ed. Reedición del vocabulario, a cargo de Guillermo Escobar Risco, como Vocabulario y phrasis en la lengua general de los indios del Perú, llamada quichua; Lima: Imprenta Rímac. 
BRAVO, C., 1986 - El tiempo de los Incas, 181 p.; Madrid: Alhambra.

BRAVO, C., 1992 - Del poder dual a la diarquía en el Estado Inca. Revista complutense de Historia de América, 18: 11-62.

CASAS, B. de las, 1948 - De las antiguas gentes del Perú, 167 p.; Lima: Librería e Imprenta D. Miranda.

CERRÓN-PALOMINO, R., 1993 - Los fragmentos de gramática del Inca Garcilaso. Lexis, XVII (2): 219-257.

CERRÓN-PALOMINO, R., 2002 - Hurin: un espejismo léxico opuesto a hanan. In: El Hombre y los Andes: Homenaje a Franklin Pease G.Y. (J. Flores \& R. Varón, eds.), 1: 219-235; Lima: Pontificia Universidad Católica del Perú.

DUVIOLS, P., 1980 - Algunas reflexiones acerca de las tesis de la estructura dual del poder incaico. Histórica, 4 (2): 183-196; Lima.

GONZALEZ HOLGUÍN, D., 1975 [1607] - Gramatica y arte nueva de la lengvua general de todo el Peru, Ilamada lengua qquichua, o lengua del Inca, 144 p.; Cabildo VaduzGeorgetown, Druck: Franz Wolf, Heppenheim a.d.B.

GONZALEZ HOLGUÍN, D., 1989 [1607] - Vocabvlario de la lengvua general de todo el Perv Ilamada lengua qquichua o del Inca, 707 p.; Lima: Universidad Nacional Mayor de San Marcos.

HERNÁNDEZ ASTETE, F., 2002 - La mujer en el Tahuantinsuyo, 168 p.; Lima: Pontificia Universidad Católica del Perú.

HERNÁNDEZ ASTETE, F., 2004 - Poder y muerte entre los incas; Madrid: Universidad Complutense de Madrid. Trabajo de Investigación Avanzado (ms.)

MOLINA, C. de., 1988 [1575?] - Relación de las fábulas y ritos de los Incas, 199 p.; Madrid: Crónicas de América 48, Historia 16.

MURÚA, M. de., 2001 [1616] - Historia general del Perú, 571 p.; Madrid: Crónicas de América, Dastin Historia.

PÄRSSINEN, M., 2003 - Tawantinsuyu. El estado inca y su organización política, 425 p.; Lima: Pontificia Universidad Católica del Perú, Instituto Francés de Estudios Andinos.

PEASE, F., 1992 - Perú Hombre e Historia. Entre el siglo XVI y el XVIII, 347 p.; Lima: EDUBANCO.

REGALADO, L., 1987 - La élite incaica frente a la crisis del Tawantinsuyu. Tesis Doctoral, Facultad de Letras y Ciencias Humanas; Lima: Pontificia Universidad Católica del Perú.

REGALADO, L., 1996a [1993] - Sucesión incaica. Aproximación al mando y poder entre los Incas a partir de la crónica de Betanzos, 133 p.; Lima: Pontificia Universidad Católica del Perú.

REGALADO, L., 1996b - Espacio andino, espacio sagrado: visión ceremonial del territorio en el período incaico. Revista Complutense de Historia de América, 22: 86-96.

ROSTWOROWSKI M., 1983 - Estructuras andinas del poder. Ideología religiosa y política, 202 p.; Lima: Instituto de Estudios Peruanos.

ROWE, J., 1985 - Probanza de los Incas nietos de conquistadores. Histórica, 9 (2): $193-$ 245; Lima.

ROWE, J., 2003 - Los Incas del Cuzco: siglos XVI-XVII-XVIII, 418 p.; Cuzco: Instituto Nacional de Cultura.

SANTO TOMAS, D., 1951 [1560] - Lexicon o vocabulario de la lengua general del Perv, 374 p.; Lima: Imprenta Santa María. 
SARMIENTO, P., 1988 [1572] - Historia de los Incas, 191 p.; Madrid: Biblioteca de viajeros Hispánicos, Miraguano Ediciones, Ediciones Polifemo.

VALCARCEL, L., 1925 - Del ayllu al imperio, 201 p.; Lima: Editorial Garcilaso.

ZIÓLKOWSKI, M., 1997 - La guerra de los wawqi: los objetivos y los mecanismos de la rivalidad dentro de la élite Inka, ss. XV-XVI, 425 p.; Quito: Ediciones ABYA-YALA.

ZUIDEMA, R., 1980 - El sistema de parentesco incaico: una nueva visión teórica. Parentesco y Matrimonio en los Andes (R. Bolton \& E. Mayer, eds.): 57-114; Lima: Pontificia Universidad Católica del Perú.

ZUIDEMA, R., 1995 [1964] - El sistema de ceques del Cuzco, 420 p.; Lima: Pontificia Universidad Católica del Perú.

ZUIDEMA, T., 2004 - La identidad de las diez panacas en el Cuzco incaico. Boletín de Arqueología de la Pontificia Universidad Católica del Perú, 8: 277-287; Lima. 